\title{
Analysis of Multi-Fingered Grasp and Manipulation of Ping-Pong Racket
}

\author{
Yuwang Liu ${ }^{1}$, a , Yuquan Leng ${ }^{1,2}$, Hongguang Wang ${ }^{1}$, Weijia Zhou ${ }^{1}$ \\ ${ }^{1}$ State Key Laboratory of Robotics, Shenyang Institute of Automation \\ Chinese Academy of Sciences, Shenyang 110016, China \\ ${ }^{2}$ Graduate School of the Chinese Academy of Sciences, Beijing 100039, China \\ aliuyuwang@sia.cn
}

Keywords: dexterous hand, grasp, manipulation, ping-pong racket

\begin{abstract}
According to the human playing of ping-pong racket, this paper originally analyzes the multi-fingered grasping and manipulating of ping-pong racket and points out the three aspects have to be studied to make sure the grasp is stable and the manipulation is dexterous. Two of those three aspects are studied respectively: the least needed number and the distribution of degrees of the multi-fingered hand is determined at first; then the kinematic planning for robotic hand grasping ping-pong racket is studied, and a new method is proposed to selected the right solution in the planning. The simulation result in the end proofs that the kinematic planning and the method are right and effective.
\end{abstract}

\section{Introduction}

With the advancement in automation and information technology, application of robotics has been spread from traditional industrial to the modern industrial, agricultural, medical, education, entertainment, aerospace, military fields and etc. As a key part of the robot, the end-effector is more and more anthropomorphic and dexterous [1-10], which is called robotic hand commonly now.

Grasp and manipulation which have been widely studied are the most important function of robotic hands [10-12]. The related literatures are almost about grasping and manipulating of globe, ellipsoid and etc, and little refer to the grasp and manipulation of hand-hold tools (such as knives, table tennis and etc.). In addition, most of the literatures about the multi-fingered grasp and manipulation research concentrate on the specific capture tasks or theoretical research based on the assumed free degrees [10-12], which does not consider physical mechanical hands.

This paper originally analyzes the multi-fingered grasp and manipulate of ping-pong racket and points out the three aspects have to be studied to make sure the grasp is stable and the manipulation is dexterous. Two of those three aspects are studied respectively: the least needed number and the distribution of degrees of the multi-fingered hand is determined at first; then the kinematic planning for robotic hand grasping ping-pong racket is studied, and a new method is proposed to selected the right solution in the planning. We put focuses on the establishment and solution of kinematics model of racket attitude, in which we propose a new method to pick the correct solution out of several potential ones.

\section{The principle of grasping racket}

When we play table tennis, the adjustment of racket position and attitude is mainly performed by the arm and the wrist respectively, the palm and fingers are mainly responsible for grasping the racket and minor change of the position and attitude of the racket. It includes grasp and manipulation when the hand uses racket. Grasp only refer to contact, without relative motion. However, manipulation includes both contact and relative motion which is a dynamic process. The main purpose of grasp and manipulation is to make the racket reach the target position and posture from the initial one, which can be realized by the specific action of fingers.

Firstly, we must ensure that the hand has enough degree of freedom, which be arranged reasonably so that it can perform the action of grasp and manipulation. Secondly, the hand could steadily hold on the racket though motion of its joint. Thirdly, it needs appropriate force between the 
hand and the racket to make sure that the racket cannot be thrown out when playing table tennis. At last, it needs to plan the motion of every joints, so that the hand in the best state. In summary, there are mainly three aspects to be studied to ensure effective grasp and manipulation:1) Determining the required freedoms to complete specific tasks, 2) Kinematics analysis to ensure stable grasp between hand and racket, and 3) Ensure that the mechanics balance of grasp and manipulation. In this paper, we just studied 1) and 2).

\section{The determination of required number of freedom}

For grasping the racket, it is necessary to analyze the required number of freedom for grasping and manipulating. We mainly depend on thumb, index and middle finger, while ring and little finger just play ancillary role when we grasp racket. So this paper assumes that the hand has three fingers, which are thumb, index and middle finger.

Take the right hand as an example. There are four contacting points between the hand and the racket, which are middle fingertip, index fingertip, thumb fingertip and palm (the middle between thumb and index finger). The racket handle and the hand palm is surface contact, while the others are point contact.

Manual thumb has five DOFs, and index and middle finger each has four DOFs. It is no doubt that robot hand can complete grasp and manipulation if the hand intends to simulate manual hand completely including the number of degrees of freedom and layout of freedom. But there will induce many problems with the increase of freedom, such as complexity increase, stability fall and so on. After a lot of analyses, we get that the hand can grasp and manipulate the racket well when the thumb finger has two 1DOF, the index finger has three 3DOF (DIP-PIP joint coupling), and the middle finger has three 1DOF, which laid as shown in Fig.1.

\section{Kinematic analysis of anthropomorphic hand}

Initially, as shown in Fig.1, index and middle finger are in the same plane which are vertical to palm, and the MP joints of the two fingers are also in the same horizontal plane. The distance between the two fingers is $m$. The atitude of the thumb in palm frame is determined by $\beta_{1}$ and $\beta_{2}$, and the position of the fingers in palm frame is determined by $k, p$ and $n$.

The three fingers are vertical with palm and every knuckle of each finger in the same line at the initial position, as shown in Fig.1. The length and joint angle of every knuckle of each finger are listed in Table 1 and shown in Fig. 1. (b).

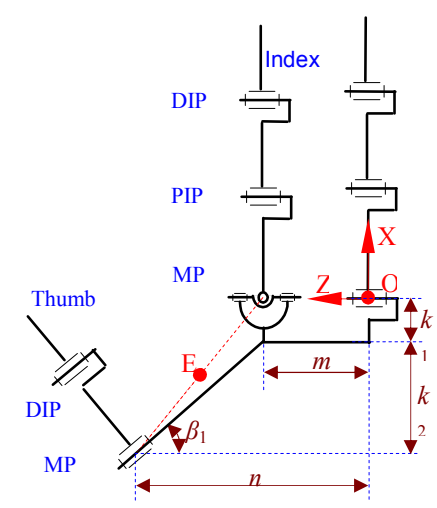

(a) initial position

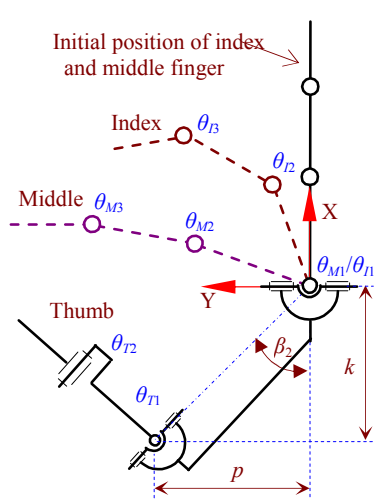

(b) parameters

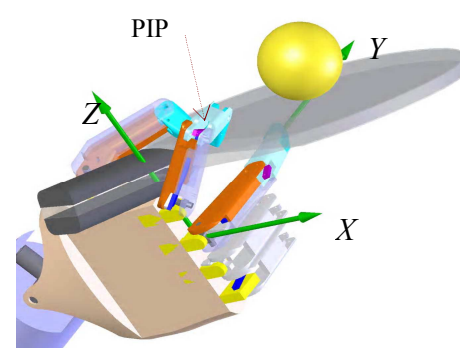

(c) model

Fig. 1. Model of the right hand 
Table 1 Parameters of the hand

\begin{tabular}{|c|c|c|c|c|c|c|c|c|}
\hline & \multicolumn{3}{|c|}{ Length of knuckle } & \multicolumn{3}{|c|}{ Angle of joint } & Parameter of Installation \\
\cline { 2 - 7 } & Under & Mid & Up & MP & PIP & DIP & Angle & Position \\
\hline Thumb & $L_{T 1}$ & & $L_{T 2}$ & $\theta_{T 1}$ & & $\theta_{T 2}$ & $\beta_{1}, \beta_{2}$ & $(-k, p, n)$ \\
\hline Index & $L_{I 1}$ & $L_{I 2}$ & $L_{I 3}$ & $\theta_{I 0}, \theta_{I 1}$ & $\theta_{I 2}$ & $\theta_{I 3}$ & & $(0,0, m)$ \\
\hline Middle & $L_{M 1}$ & $L_{M 2}$ & $L_{M 3}$ & $\theta_{M 1}$ & $\theta_{M 2}$ & $\theta_{M 3}$ & & $(0,0,0)$ \\
\hline
\end{tabular}

The distance between the two MPs of the thumb and the middle finger along the axis is $k$, and $k=k_{1}+k_{2}$, in which $k_{1}$ and $k_{2}$ are shown in Fig 1 . (a). Then we can get $k_{2}=(n-m) / \tan \beta_{1}$, in which $\beta_{1}$ and $\beta_{2}$ are the installed angles of the thumb. $\beta_{1}$ is the angle between the base of the thumb and the horizontal plane, $\beta_{2}$ is the angle between the axis of the thumb MP and the vertical plane, so $\tan \beta_{2}=p \sin \beta_{1} / k_{2}$. As shown in Fig.1(c), the base coordinate system is built on the center of the middle finger's MP joint, whose Z-axis points to index finger MP joint, $\mathrm{Y}$-axis is perpendicular to the palmand, $\mathrm{X}$-axis is determined by the right-hand rule. The middle finger structure (1-DOF) lies on the $\mathrm{XY}$ plane, the coordinates of index finger's MP joint is $(0,0, m)$ and the position of the thumb finger MP joint can be dertermined by $(-k, p, n)$.

The kinematics of each finger in their own coordinates system can be calculated, and then be converted to the basic coordinate system. Each finger's coordinates system is defined as follows: $\mathrm{Z}$-axis is along the MP joint flexion / extension direction, $\mathrm{X}$-axis along the center line of the initial position of the fingers, and $\mathrm{Y}$-axis pointing to finger flexion / extension direction, as shown in Fig.1(c). The coordinates system of thumb finger can be defined with reference to the above.

The position of the end points of the middle finger, the index finger and the thumb finger are expressed as $\left[x_{M}^{*}, y_{M}^{*}, z_{M}^{*}\right]^{T},\left[x_{I}^{*}, y_{I}^{*}, z_{I}^{*}\right]^{T}$ and $\left[x_{T}^{*}, y_{T}^{*}, z_{T}^{*}\right]^{T}$ in their own coordinate system, which can be got by the analysis of kinematics of the finger.

We can then get the kinematic of each finger in the basic coordinate system.

To the index finger, the position of the end point in the basic coordinate is the same with that in its own coordinate, so

$$
\left[x_{M}, y_{M}, z_{M}\right]^{T}=\left[x_{M}^{*}, y_{M}^{*}, z_{M}^{*}\right]^{T}
$$

To the index finger, the position of the end point in the basic coordinate system can be expressed

as

$$
\left[x_{I}, y_{I}, z_{I}\right]^{T}=\left[x_{I}^{*}, y_{I}^{*}, z_{I}^{*}\right]^{T} \operatorname{Rot}\left(Y,-\theta_{I 0}\right) \operatorname{Trans}(Z, m)
$$

To the thumb finger, the position of the end point in the basic coordinate system can be expressed

as

$$
\left[x_{T}, y_{T}, z_{T}\right]^{T}=\left[x_{T}^{*}, y_{T}^{*}, z_{T}^{*}\right]^{T} \operatorname{Trans}(Z, m) \operatorname{Trans}\left(X,-k_{1}\right) \operatorname{Rot}\left(Y,-\beta_{1}\right) \operatorname{Rot}\left(X,-\beta_{2}\right) \operatorname{Trans}\left(Z, p / \sin \beta_{2}\right)
$$

\section{Analysis of the model and constraints of the motion of the racket}

As stated above, the grasping of racket can be seen as four-point contact, and the four contact points can be named as A, B, C, and E, which shape a tetrahedron,as shown in Fig 2. The racket motion can be determined through the position of two different surface lines $\mathrm{AB}$ and $\mathrm{EC}$. The distance between the two lines is the racket thickness $d$, and $\mathrm{AB}$ is always above the $\mathrm{EC}$. The point $\mathrm{E}$ is the projection of the point $\mathrm{D}$, which is the midpoint of $\mathrm{MN}$, and point $\mathrm{N}$ is the center of the MP joint of the index finger and point $\mathrm{M}$ is the center of the MP joint of the thumb finger. Moreover, the point $\mathrm{C}$ lies on the centerline of the reverse surface of the racket. As the middle finger has only one freedom, so the position of $\mathrm{C}$ and $\mathrm{E}$ can be determined. Then we can plan racket gesture depending on point $\mathrm{A}$ and point $\mathrm{B}$. The method is to ensure the point $\mathrm{A}$ on the racket surface firstly and then plan the motion of 3DOF joints of index finger to let the endpoint of the index finger on the racket surface.

As shown in Fig 2. (c), point $M$ is the center of the MP joint of the thumb finger, and its coordinate is $(-k, p, n)$. Point $\mathrm{N}$ is the center of the MP joint of the index finger, and its coordinate is $(0$, $0, m)$, and point $\mathrm{E}$ is the midpoint of the line $M N$, so we can obtain its coordinate $\left(x_{E}, y_{E}, z_{E}\right)=\left(\left(x_{M}+x_{N}\right) / 2,\left(y_{M}+y_{N}\right) / 2,\left(z_{M}+z_{N}\right) / 2\right)$ The coordinates of $\mathrm{C}, \mathrm{A}$ and $\mathrm{B}$ are $\left(x_{C}, y_{C}, z_{C}\right)=\left(x_{M}, y_{M}, z_{M}\right),\left(x_{A}, y_{A}, z_{A}\right)=\left(x_{T}, y_{T}, z_{T}\right)$ and $\left(x_{B}, y_{B}, z_{B}\right)=\left(x_{I}, y_{I}, z_{I}\right)$ respectively. 
The purpose of kinematic planning is to obtain the rotation angle of every joint of each finger so that hand can fully contact with the table racket, and to achieve the desired racket motion to hit the ball. Besides the kinematics analysis of multi-fingered hand, we also need to define the line EC, the attitude of the racket, the surface equation of the racket, mathematical model of manipulation and so on.

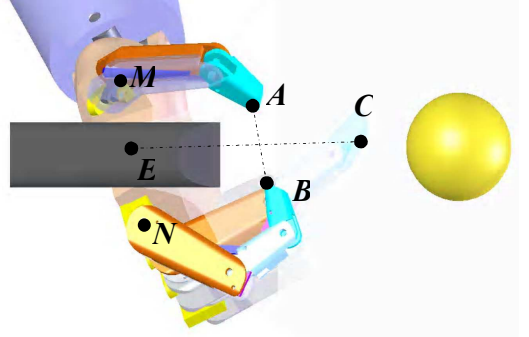

(a)Four contact points

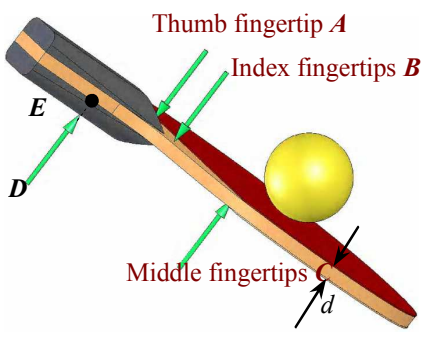

(b)The position of the points

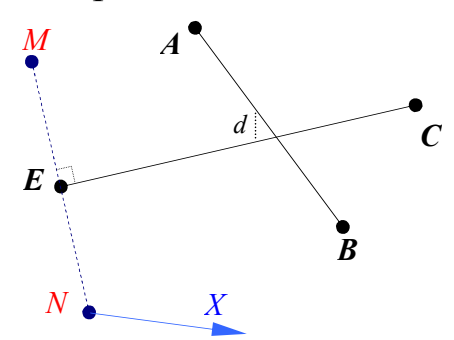

(c)Schematic

Fig. 2. The contact points

According to human grasping on racket, assuming the angle between $\mathrm{EC}$ and $\mathrm{MN}$ is $\varphi$, so $\varphi=\arccos \left(\mathbf{v}_{E C} \cdot \mathbf{v}_{M N} /\left|\mathbf{v}_{E C} \| \mathbf{v}_{M N}\right|\right)$

In the equation,

$\mathbf{v}_{E C}=\left\{x_{C}-x_{E}, y_{C}-y_{E}, z_{C}-z_{E}\right\} \mathbf{v}_{M N}=\left\{x_{M}-x_{N}, y_{M}-y_{N}, z_{M}-z_{N}\right\}$

Puting the coordinates of the point $\mathrm{E}$, that of the endpoint of the thumb finger, and equation (1) into equation (4), we can obtain the driving angle of the middle finger $\theta_{M 1}$ and the position of $\mathrm{C}\left(x_{C}, y_{C}\right.$ , $\left.z_{C}\right)$.

At the initial time, EC is on the reverse of the racket and MN parallels to the racket plane. $\mathrm{E}$ is the midpoint of $\mathrm{MN}$, so the initial posture of the racket can be deterimined by the points $\mathrm{M}, \mathrm{N}$ and $\mathrm{C}$. The movement of the racket is equal to the plane $\mathrm{MNC}$ rotating around $\mathbf{v}_{E C}$. According to the angle $\psi \in(-\pi / 2, \pi / 2)$, we can calculate $\mathbf{v}_{p}$ which is a vector vertical to the racket surface, also EC is on the reverse of the racket, so we can obtain the equation of the reverse of the racket.

From the above analysis, the equation of the reverse of the racket on the initial moment can be expressed as

$$
a\left(x-x_{C}\right)+b\left(y-y_{C}\right)+c\left(z-z_{C}\right)=0
$$

where $a=\left[\begin{array}{ll}y_{M}-y_{C} & z_{M}-z_{C} \\ y_{N}-y_{C} & z_{N}-z_{C}\end{array}\right] \quad b=\left[\begin{array}{cc}z_{M}-z_{C} & x_{M}-x_{C} \\ z_{N}-z_{C} & x_{N}-x_{C}\end{array}\right] \quad c=\left[\begin{array}{ll}x_{M}-x_{C} & y_{M}-y_{C} \\ x_{N}-x_{C} & y_{N}-y_{C}\end{array}\right]$.

From which, we can get the normal vector of the initial posture of the racket surface $\mathbf{n}_{1}=\{a, b, c\}$. After EC rotates an angle $\psi(\psi \neq 0)$, assume the normal vector of the racket is $\mathbf{n}_{2}=\{P, Q, R\}$, then

$$
\left(\mathbf{v}_{E C} \cdot \mathbf{n}_{2}=0\right) \&\left(\cos \psi=\mathbf{n}_{1} \cdot \mathbf{n}_{2} /\left|\mathbf{n}_{1} \| \mathbf{n}_{2}\right|\right)
$$

In the equation

$$
\left\{\begin{array}{l}
\mathbf{n}_{2}=\{P, Q, R\}=\left\{k Q, Q, Q \sqrt{k^{2}+1} / \tan \psi\right\}=\left\{k, 1, \sqrt{k^{2}+1} / \tan \psi\right\} \\
k=\left(X Y \tan ^{2} \psi \pm Z \sqrt{X^{2} \tan ^{2} \psi+Y^{2} \tan ^{2} \psi+Z^{2}}\right) /\left(X^{2} \tan ^{2} \psi-Z\right) \\
X=x_{C}-x_{E} \quad Y=y_{C}-y_{E} \quad Z=z_{C}-z_{E}
\end{array}\right.
$$

The two surface of racket are parallel, so we can get the equation of obverse

$$
k x+y+z \sqrt{k^{2}+1} / \tan \psi+D_{2}=0
$$

In the equation, $D_{1}=-k x_{C}-y_{C}-z_{C} \sqrt{k^{2}+1} / \tan \psi$ and $d=\left(D_{2}-D_{1}\right) / \sqrt{k^{2}+1+\left(\sqrt{k^{2}+1} / \tan \psi\right)^{2}}$.

As a result, we can get the equation of the surface of the racket.

As stated above, the grasp and manipulation involves four contact points (shown in Fig.2), in which $\mathrm{E}$ and $\mathrm{C}$ has been determined. The task of kinematic plan is to decide the movement of each joint of thumb and index finger, so that the fingertips is always on the surface of racket. When $\Psi$ is known, kinematic planning is to solve the angle movemont of the 1DOF thumb and that of the 3DOF index . 
By puting the coordinates of point $\mathrm{A}$ and $\mathrm{B}$ calculated above into the plane equation (6), we can verify whether the coordinates of $\mathrm{A}$ and $\mathrm{B}$ meets the equation. If met, then the points are on the plane. Another method is to calculate the distance between A or B and the upper surface of the racket. If the distance is zero, then the point is on the plane.The distance can be calculated by following equations.

$$
\left\{\begin{array}{l}
d_{A}=\left|k x_{A}+y_{A}+\left(\frac{\sqrt{k^{2}+1}}{\tan \psi}\right) z_{A}+D_{2}\right| / \sqrt{k^{2}+1+\left(\frac{\sqrt{k^{2}+1}}{\tan \psi}\right)^{2}} \\
d_{B}=\left|k x_{B}+y_{B}+\left(\frac{\sqrt{k^{2}+1}}{\tan \psi}\right) z_{B}+D_{2}\right| / \sqrt{k^{2}+1+\left(\frac{\sqrt{k^{2}+1}}{\tan \psi}\right)^{2}}
\end{array}\right.
$$

In the above analysis, the angle of the finger joint is limited in certain range, considering the action of the human hand and mechanical constraints. In addition, in order to ensure the fingertips touch the surface of the racket, some angle value in the range is not infeasible solutions, so we need to add specific constraints to make the solution is in the feasible region.

According to the human hand, the range of the tuning angle of mechanical hand joints is $\left[0^{\circ}, 90^{\circ}\right]$. In order to make sure that thumb can exert propose force on the surface of racket, we should keep $\theta_{T 1}+\theta_{T 2} \in\left[60^{\circ}, 120^{\circ}\right]$. In order to make sure that the middle fingertip make more anthropomorphic, it is best to keep $\theta_{M 1}+\theta_{M 2}+\theta_{M 3} \in\left[30^{\circ}, 90^{\circ}\right]$.

It must ensure that the up-knuckle and mid- knuckle of the index finger (or PIP joint and DIP joint) are on the top of the racket so as to let the index finger exert force on the racket. In addition, the position of the thumb and middle finger has only one solution, because they each has one freedom. That is, we only need to limit one of them (PIP joint and DIP joint) on the top of the racket. Here we select the PIP joint as an example.

According to (24), choose any three points on the surface of the racket, $S_{1}, S_{2}$ and $S_{3}$, and P means PIP joint. At initial time, the position of of $\mathrm{P}$ is $\left(l_{I 1}, 0, m\right)$. The end of time, it is $\left[l_{I 1}, 0, m\right] \operatorname{Rot}\left(Z, \theta_{I 1}\right) \operatorname{Rot}\left(Y,-\theta_{I 0}\right)$. The volume of the tetrahedron $S_{1} S_{2} S_{3} P$ can be expressed as

$$
V_{E S_{1} S_{2} S_{3}}=\frac{1}{6}\left|\begin{array}{llll}
x_{S_{1}} & y_{S_{1}} & z_{S_{1}} & 1 \\
x_{S_{2}} & y_{S_{2}} & z_{S_{2}} & 1 \\
x_{S_{3}} & y_{S_{3}} & z_{S_{3}} & 1 \\
x_{P} & y_{P} & z_{P} & 1
\end{array}\right|
$$

Assuming the positive direction of plane $S_{1} S_{2} S_{3}$ is $\overrightarrow{S_{3} S_{1}} \times \overrightarrow{S_{2} S_{1}}$, if the point $\mathrm{P}$ is above the plane, then $V_{E S S_{S} S_{3}}>0$, if $\mathrm{P}$ below the plane, then $V_{E S S_{S} S_{3}}<0$, if the point $\mathrm{P}$ in the plane, then $V_{E S S_{S} S_{3}}=0$. The task of grasping the racket requires $V_{E S S_{2} S_{3}}>0$.

\section{Example}

According to the human hand, assuming $m=25 \mathrm{~mm}, n=70 \mathrm{~mm}, \beta_{1}=30^{\circ}, \beta_{2}=45^{\circ}, k_{1}=5 \mathrm{~mm}$. we can get $k$ and $p$ depending on equations deduced in section" Kinematic analysis of anthropomorphic hand", and get movement relationship of coupling joint, that is $\theta_{M 2}=\theta_{M 1}, \theta_{M 3}=0.1 \theta_{M 1}, \quad \theta_{I 2}=\theta_{I 3}, \quad \theta_{T 1}=\theta_{T 2}$. The other parameters and the range of joints are given in Table 2.

Assuming the tuning angle of the racket is zero, then $\psi=5^{\circ}$; assuming the racket's thickness $d=10 \mathrm{~mm}$, the coefficient of friction is $\mu_{A}=\mu_{B}=\mu_{C}=\mu_{E}=0.1$ and the acceleration is $\mathbf{a}=\mathbf{0}$.

Assuming $\theta_{T 1}=\theta_{T 2} \in\left[10^{\circ}, 40^{\circ}\right]$ and $\theta_{M 1}=\theta_{M 2}=\theta_{M 3} \in\left[10^{\circ}, 45^{\circ}\right]$ in the simulation, in the range $\theta_{I 0} \in$ $\left[-25^{\circ}, 25^{\circ}\right], \theta_{I 1} \in\left[10^{\circ}, 40^{\circ}\right]$ and $\theta_{I 2}=\theta_{I 3} \in\left[20^{\circ}, 90^{\circ}\right]$, we can get the position of the end point of the index finger. Then we can get the feasible region of the angle of each index finger joint, as shown in Fig.3, which shows that when the index finger adduct / abduct to the middle finger, it should contact the racket face, and as the adduction / abduction angle become greater, the feasible region of $\theta_{I 1}$ and $\theta_{I 2}$ become larger. When $\theta_{I 0}<0.28 \mathrm{rad}$, the index fingertip is impossible to contact the racket. When $\theta_{I 0}$ is constant, the feasible region of $\theta_{I 2}$ becomes smaller as $\theta_{I 1}$ becomes lager. Especially, there is only one solution when $\theta_{I 1}=0.58 \mathrm{rad}$. 
Table 2 Specific parameters

\begin{tabular}{|c|c|c|c|c|c|c|}
\hline & \multicolumn{3}{|c|}{$\begin{array}{c}\text { Length of } \\
\text { knuckle(mm) }\end{array}$} & \multicolumn{3}{c|}{ Angle of joint $\left(^{\circ}\right)$} \\
\cline { 2 - 7 } & Under & Mid & Up & MP & PIP & DIP \\
\hline Thumb & $L_{T 1}=50$ & & $L_{T 2}=30$ & $\theta_{T 1}=40$ & & $\theta_{T 2}=40$ \\
\hline Index & $L_{I 1}=50$ & $L_{I 2}=30$ & $L_{I 3}=25$ & $\theta_{I 0} \in[-25,25]$ & $\theta_{I 2} \in$ & $\theta_{I 3} \in$ \\
& & & $\theta_{I 1} \in[10,40]$ \\
{$[20,90]$} & {$[20,90]$} \\
\hline Middle & $L_{M 1}=50$ & $L_{M 2}=30$ & $L_{M 3}=25$ & $\theta_{M 1}$ & $\theta_{M 2}$ & $\theta_{M 3}$ \\
\hline
\end{tabular}

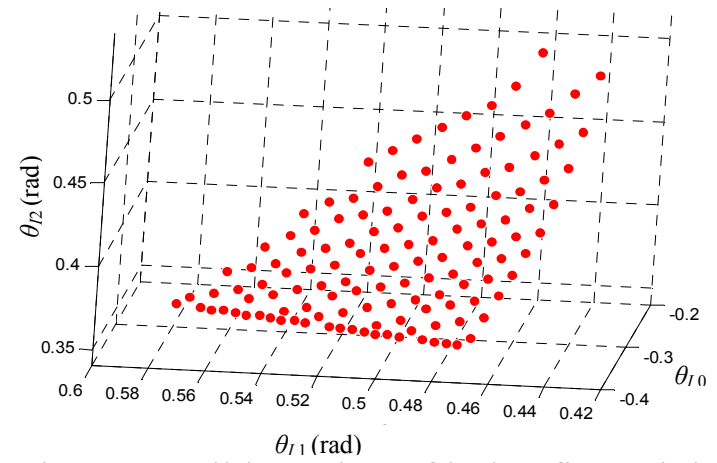

Fig. 3. Feasible region of index finger joint

\section{Conclusion}

(1) According to the method that human grasping on ping-pong racket, this paper at the first time systematically analyzes and researches the multi-fingered grasping on ping-pong racket.

(2) Based on the task of playing racket, this paper firstly analyze the least DOF of hand so that to perform stable grasp and manipulation, and then design the robotic hand referencing to the structure of human hand.

(3) Considering the arrangement of the contact points, thickness of the racket and contact friction and other important factors, this paper establishes the geometric and mathematical models of grasp and manipulation of ping-pang racket, and then researches the kinematics planning.

(4) A method using positive and negative value of tetrahedron volume to obtain the correct solution from multiple solutions of kinematics is proposed.

\section{References}

[1] MASON. M. T, SALISBURY.J. K. Robot hands and the mechanics of manipulation [M]. MIT Press, Cambridge, USA, 1985, pp. 3-93.

[2] BIAGIOTTI. L, LOTTI. F, MELCHIORR. Ci, VASSURA.G.. Design aspects for advanced robot hands [J]. 2002 IEEE/RSJ International Conference on Intelligent Robots and Systems, 2002:1-39.

[3] PONS. J. L, CERES. R, PFEIFFER. F. Multi-fingered dextrous robotics hand design and control: a review [J]. Robotica, 1999, 17:661-674.

[4] PETER. J, KYBERD, LIGHT Colin, PAUL. H, CHAPPELL. The design of anthropomorphic prosthetic hands: A study of the Southampton Hand [J]. Robotica, 2001, 19:593-600.

[5] JACOBSEN. S. C, WOOD. J. E, KNUTTI. D. F. The UTAH/M IT dexterous hand: work in progress [J]. The International Journal of Robotics Research, 1984, 4: 21-50.

[6] EUSEBI. A, FANTUZZI.C, MELCHIORRI. C, SANDRI. M, TONIELL. A. The U.B. hand II control system: design features and experimental results [J]. IEEE, 1994.

[7] LOVCHIK. C. S, DIFTLER. M. A. The robonaut hand: a dexterous robot hand for space [J]. Proceedings of the 1999 IEEE International Conference on Robotics \& Automation, 1999: 907-912.

[8] DECHEV. N, CLEGHORN. W. L, NAUMANN. S. Multiple finger passive adaptive grasp prosthetic hand [J]. Mechanism and Machine Theory, 2001, 36: 1157-1173.

[9] RICHARD. M, Murray, LI Zexiang, SHANKAR. Sastry. A Mathematical Introduction to Robotic Manipulation [M]. CRC Press, Inc., 2000 Corporate Blvd., N. W., Boca Ration, Florida 33431, USA.

[10] Stappen A F V, Wentink C, Overmars M H. Computing Immobilizing Grasps of Polygonal Parts. Int .J. of Robotics Research. 2006,19 (5) : 467-479.

[11]Brost R C, Goldberg K Y. A Complete Algorithm for Designing Planar Fix Tures Using Modular Components. IEEE Transactions on Robots and Automation, 1996,12 (1) : 31-46.

[12]XIONG Youlun, YIN Zhouping, XIONG Caihua. Robotic Manipulation [M]. Hubei Science and Technology Press, Wuhan, 2002.01 